\title{
Profiles of off-diagonal Components of Static Linear and Nonlinear Polar- izabilities of Doped Quantum Dots Driven by Gaussian White Noise
}

\author{
Surajit Saha ${ }^{\mathrm{a}}$, Jayanta Ganguly ${ }^{\mathrm{b}}$ and Manas Ghosh ${ }^{\mathrm{c}, *}$ \\ ${ }^{a}$ Department of Chemistry, Bishnupur Ramananda College, Bishnupur, Bankura 722122, West Bengal, India \\ ${ }^{b}$ Department of Chemistry, Brahmankhanda Basapara High School, Basapara, Birbhum 731215, West Bengal, India \\ ${ }^{c}$ Department of Chemistry, Physical Chemistry Section, Visva Bharati University, Santiniketan, Birbhum 731235, West \\ Bengal, India
}

\begin{abstract}
We investigate the profiles of off-diagonal components of static linear $\left(\alpha_{x y}, \alpha_{y x}\right)$, first nonlinear $\left(\beta_{x y y}, \beta_{y x x}\right)$, and second nonlinear $\left(\gamma_{x x y y}\right.$ and $\left.\gamma_{y y x x}\right)$, polarizabilities of repulsive impurity doped quantum dots. The dopant impurity potential is expressed as a Gaussian function. The study thrusts on investigating the role of Gaussian white noise on the polarizability components. The noise has been applied additively and multiplicatively to the system (in Stratonovich sense). The doped system is further subjected to a static external electric field. The dopant site and the mode of application of noise design the polarizability components in a subtle manner. We have found that the strength of additive noise fails to influence the polarizability components. However, the multiplicative noise introduces greater delicacy in the observed profiles of polarizability components. The present study reveals appearance of maximization and saturation in the linear and nonlinear polarizability components on some occasions which bears substantial technological importance in the field of noisedriven optical properties of doped quantum dot systems.
\end{abstract}

Keywords: Quantum dot, impurity, Gaussian white noise, static polarizability, off-diagonal components, dopant location.

\section{INTRODUCTION}

Quantum dots (QDs) are the destinations we can finally arrive so far, as miniaturization of semiconductor devices is concerned. QDs are familiar for displaying much more rich nonlinear optical effects than the bulk materials. Thus, they have undergone extensive applications as an indispensable ingredient in a variety of optical devices. Rigorous study of optical properties of these devices endows us with lots of important information about their energy spectrum, the Fermi surface of electrons, and the value of electronic effective mass. These features have made QDs widely recognized high-performance semiconductor optoelectronic materials. However, QDs are often contaminated with dopants during their fabrication which abruptly alter their properties. The said contamination introduces additional potential to the QD system which invariably undergoes interaction with intrinsic QD confinement potential. The interaction appears to be responsible for the dramatic change in various properties of QD. A large number of investigations on doped QD [1-7] therefore accrue with increasing need of exploring their properties. Within the realm of optoelectronic applications, impurity guided modulation of linear and nonlinear optical properties have been found to be immensely important in

*Address correspondence to this author at the Department of Chemistry, Physical Chemistry Section, Visva Bharati University, Santiniketan, Birbhum 731235, West Bengal, India; Tel: (+91) (3463)261526, (3463)262751-6 (Ext.467); Fax: +91 3463 262672;

E-mail: pcmg77@ rediffmail.com photodetectors and in several high-speed electro-optical devices $[8,9]$. A cornucopia of important works on both linear and nonlinear optical properties of these structures was therefore an anticipated outcome [8, 10-22].

External electric field has often been found to illuminate important aspects related with concerned impurities. The electric field changes the energy spectrum of the carrier and controls the performance of the optoelectronic devices. Moreover, the electric field often hampers the symmetry of the system and facilitates the emergence of nonlinear optical properties. Thus, the applied electric field assumes special attention in view of understanding the optical properties of doped QDs [23-30].

Recently we have amply discussed the importance of noise [31-33] in influencing the performances of QD devices. In these works we have explored the role of Gaussian white noise on the diagonal components of frequencydependent linear [31], first nonlinear [32], and the third nonlinear [33] polarizabilities of doped QD. In the present manuscript we explore the role of Gaussian white noise on the off-diagonal components of static linear $\left(\alpha_{x y}, \alpha_{y x}\right)$, first nonlinear (second order) $\left(\beta_{x y y}, \beta_{y x x}\right)$, and the second nonlinear (third order $)\left(\gamma_{x x y y}, \gamma_{y y x x}\right)$, polarizabilities of doped QD. Investigation on off-diagonal components demands exploration as they interact differently with the applied field from their diagonal analogs and thus expected to exhibit noticeably distinct features. Of late Sahin made some important contribution to the third order optical property of a spherical QD and analyzed the role of impurity [13]. The notable work of 
Karabulut and Baskoutas [20] also deserves mention in a similar context which includes the effect of electric field and impurity. In the present study noise has been applied to the system additively and multiplicatively [31-33]. An external electric field of given intensity has been applied to the doped system which acts as a perturbation and generates linear and nonlinear responses. We have put special emphasis on the role of dopant location and the noise characteristics as they happen to modulate the static off-diagonal polarizability components. The role of dopant site has been critically explored because of its well-known influence in modulating the optical properties of doped heterostructures. In their previous works Karabulut and Baskoutas [20], and Baskoutas et al. [23] highlighted the importance of off-centre impurities and introduced a novel numerical method (PMM; potential morphing method). The present analysis reveals the nuances in the profiles of aforesaid polarizability components as a result of intricate interplay between noise characteristics and the effective confinement potential of the doped QD system. The effective confinement potential has a strong dependence on the site of dopant incorporation and thus the latter makes a significant contribution in the fabrication of the overall pattern of the polarizability components. The significance of mode of application of noise (additive/ multiplicative) to the doped system has also been thoroughly addressed in the present manuscript.

\section{METHOD}

Our model Hamiltonian represents a 2-d quantum dot with single carrier electron laterally confined (parabolic) in the $\mathrm{x}-\mathrm{y}$ plane. The confinement potential reads $V(x, y)=$ $\frac{1}{2} m * \omega_{0}^{2}\left(x^{2}+y^{2}\right)$, where $\omega_{0}$ is the harmonic confinement frequency. The parabolic confinement potential has found extensive usage in various studies on QDs [1, 3, 4, 6, 18, 24], particularly in the study of optical properties of doped QDs by Çakir et al. [14]. A perpendicular magnetic field (B $\sim \mathrm{mT}$ in the present work) is also present as an additional confinement. Using the effective mass approximation we can write the Hamiltonian of the system as

$$
H_{0}^{\prime}=\frac{1}{2 m^{*}}\left[-i \hbar \nabla+\frac{e}{c} A\right]^{2}+\frac{1}{2} m^{*} \omega_{0}^{2}\left(x^{2}+y^{2}\right)
$$

In the above equation $\mathrm{m}^{*}$ stands for the effective electronic mass within the lattice of the material. The value of $\mathrm{m}^{*}$ has been chosen to be $0.067 \mathrm{~m}_{0}$ representing GaAs quantum dots.

We have set $\hbar=e=m_{0}=a_{0}=1$ and performed our calculations in atomic unit. In Landau gauge $\left[\mathrm{A}=\left(B_{y}, 0,0\right)\right]$ (A being the vector potential), the Hamiltonian transforms to

$$
\begin{aligned}
& H_{0}^{\prime}=-\frac{\hbar^{2}}{2 m^{*}}\left(\frac{\delta^{2}}{\delta x^{2}}+\frac{\delta^{2}}{\delta y^{2}}\right)+\frac{1}{2} m^{*} \omega_{0}^{2} x^{2}+\frac{1}{2} m^{*}\left(\omega_{0}^{2}+\right. \\
& \left.\omega_{c}^{2}\right) y^{2}-i \hbar \omega_{c} y \frac{\delta}{\delta x}
\end{aligned}
$$

$\omega_{c}=\frac{e B}{m^{*} c}$ being the cyclotron frequency. $\Omega^{2}=\omega_{0}^{2}+\omega_{c}^{2}$ can be viewed as the effective frequency in the y-direction.
We now introduce impurity (dopant) to QD and the dopant is represented by a Gaussian potential [34-36]. To be specific, in the present case we write the impurity potential as

$V_{i m p}=V_{0} e^{-\xi\left[\left(x-x_{0}\right)^{2}+\left(y-y_{0}\right)^{2}\right]}$. The choice of positive values for $\xi$ and $\mathrm{V}_{0}$ gives rise to repulsive impurity. Among various parameters of impurity potential, $\left(\mathrm{x}_{0}, \mathrm{y}_{0}\right)$ denotes the dopant coordinate, $\mathrm{V}_{0}$ is a measure of strength of impurity potential, and $\xi^{-1}$ determines the spatial stretch of impurity potential. Recently Khordad and his co-workers introduced a new type of confinement potential for spherical QDs called Modified Gaussian Potential, MGP [37, 38]. The Hamiltonian of the doped system reads

$$
H_{0}=H_{0}^{\prime}+V_{\text {imp }} \text {. }
$$

We have employed a variational recipe to solve the timeindependent Schrödinger equation and the trial function $\psi(x, y)$ has been constructed as a superposition of the product of harmonic oscillator eigenfunctions [31-33] $\phi_{n}(p x)$ and $\phi_{m}(q y)$ respectively, as

$$
\psi(x, y)=\sum_{n, m} C_{n, m} \phi_{n}(p x) \phi_{m}(q y)
$$

Where $C_{n, m}$ are the variational parameters and $\mathrm{p}=\sqrt{\frac{m^{*} \omega_{0}}{\hbar}}$ and $\mathrm{q}=\sqrt{\frac{m^{*} \Omega}{\hbar}}$. The general expression for the matrix elements of $H_{0}^{\prime}$ and $V_{i m p}$ in the chosen basis has been derived [31 - 33]. In the linear variational calculation, the requisite number of basis functions has been exploited after performing the convergence test. And $\mathrm{H}_{0}$ is diagonalized in the direct product basis of harmonic oscillator eigenfunctions.

With the application of noise the time-dependent Hamiltonian becomes

$$
H(t)=H_{0}+V_{1}(t)
$$

The noise consists of random term $(\sigma(t))$ which follows a Gaussian distribution (produced by Box-Muller algorithm) having strength $\mu$. It is characterized by the equations [3133]:

$<\sigma(t)>=0$,

the zero average condition, and

$$
<\sigma(t) \sigma\left(t^{\prime}\right)>=2 \mu \delta\left(t-t^{\prime}\right),
$$

the two-time correlation condition where the correlation time is negligible. The Gaussian white noise has been administered additively $\left[V_{1}(t)=\sigma(t)\right]$ as well as multiplicatively $\left[V_{1}(t)=\sigma(t)(x+y)\right][31-33]$.

The external static electric field $V_{2}$ of strength $\varepsilon$ is now applied where

$$
V_{2}=\varepsilon_{x} \cdot x+\varepsilon_{y} \cdot y \text {. }
$$

Where $\varepsilon_{x}$ and $\varepsilon_{y}$ are the field intensities along the $x$ and the $y$ directions. Now the time-dependent Hamiltonian reads

$$
H(t)=H_{0}+V_{1}(t)+V_{2} .
$$


The matrix elements due to $V_{1}(t)$ and $V_{2}$ can be readily derived [31-33].

The evolving wave function can now be expressed by a superposition of the eigenstates of $H_{0}$, i.e.

$$
\psi(x, y, t)=\sum_{q} a_{q}(t) \psi_{q}
$$

The associated time-dependent Schrödinger equation (TDSE) has now been solved numerically to obtain $\psi(x, y, t)$. For the numerical solution we have invoked 6th order Runge-Kutta-Fehlberg method with a time step size $\Delta t=0.01$ a.u. on verifying the numerical stability of the integrator. The time-dependent superposition coefficients $\left[a_{q}(t)\right]$ has been used to calculate the time-average energy of the dot $\langle E\rangle$ [31-33]. We have determined the energy eigenvalues for various combinations of $\varepsilon_{x}$ and $\varepsilon_{y}$ and used them to compute some of the off-diagonal components of linear and nonlinear polarizabilities by the following relations obtained by numerical differentiation. For linear polarizability:

$$
\begin{aligned}
& \alpha_{x y} \varepsilon_{x} \varepsilon_{y}= \\
& \frac{1}{48}\left[E\left(2 \varepsilon_{x}, 2 \varepsilon_{y}\right)-E\left(2 \varepsilon_{x},-2 \varepsilon_{y}\right)-E\left(-2 \varepsilon_{x}, 2 \varepsilon_{y}\right)+\right. \\
& \left.E\left(-2 \varepsilon_{x},-2 \varepsilon_{y}\right)\right]-\frac{1}{3}\left[E\left(\varepsilon_{x}, \varepsilon_{y}\right)-E\left(\varepsilon_{x},-\varepsilon_{y}\right)-\right. \\
& \left.E\left(-\varepsilon_{x}, \varepsilon_{y}\right)+E\left(-\varepsilon_{x},-\varepsilon_{y}\right)\right]
\end{aligned}
$$

And a similar expression is used for computing $\alpha_{y x}$ component.

The off-diagonal components of first non-linear polarizability (second order/quadratic hyperpolarizability) are calculated from following expressions.

$$
\begin{aligned}
& \beta_{x y y} \varepsilon_{x} \varepsilon_{y}^{2}=\frac{1}{2}\left[E\left(-\varepsilon_{x},-\varepsilon_{y}\right)-E\left(\varepsilon_{x}, \varepsilon_{y}\right)+E\left(-\varepsilon_{x}, \varepsilon_{y}\right)-\right. \\
& \left.E\left(\varepsilon_{x},-\varepsilon_{y}\right)\right]+\left[E\left(\varepsilon_{x}, 0\right)-E\left(-\varepsilon_{x}, 0\right)\right]
\end{aligned}
$$

And a similar expression is used for computing $\beta_{y x x}$ component.

The off-diagonal components of second nonlinear polarizability (third order/cubic hyperpolarizability) are given by

$$
\begin{aligned}
& \gamma_{x x y y} \varepsilon_{x}^{2} \varepsilon_{y}^{2}=2\left[E\left(\varepsilon_{x}\right)+E\left(-\varepsilon_{x}\right)\right]+2\left[E\left(\varepsilon_{y}\right)+E\left(-\varepsilon_{y}\right)\right]- \\
& {\left[E\left(\varepsilon_{x}, \varepsilon_{y}\right)+E\left(-\varepsilon_{x},-\varepsilon_{y}\right)+E\left(\varepsilon_{x},-\varepsilon_{y}\right)+\right.} \\
& \left.E\left(-\varepsilon_{x}, \varepsilon_{y}\right)\right]-4 E(0)
\end{aligned}
$$

And a similar expression is used for computing $\gamma_{y y x x}$ component.

\section{RESULTS AND DISCUSSION}

\section{A. Role of Dopant Location}

Fig. (1a and 1b) depict the profiles of $\alpha_{x y}$ as a function of dopant location $r_{0}$ for additive and multiplicative noise, respectively. In case of additive noise the said off-diagonal component remains nearly static with $\mathrm{r}_{0}$ up to $r_{0} \sim 20.0$ a.u. As the dopant is moved further, the component makes a distinct jump and finally at $r_{0} \sim 45.0$ a.u. it begins to settle with further shift of dopant (Fig. 1a). The sharp jump in the linear off-diagonal components can be attributed to sudden fall in the dot confinement beyond a dopant location of 20.0 a.u. Additive noise exploits this lack of confinement and increases the dispersive character of the system as evident from the prominent jump of said polarizability component. The saturation appearing at $r_{0} \sim 45.0$ a.u. indicates a balanced situation between the effective confinement and the noise strength. Similar profile exhibits quite different appearance when multiplicative noise is applied to the system. The profile now displays prominent maxima at $r_{0} \sim 20.0$ a.u. Fig. (1b). A dopant located in the vicinity of dot confinement centre simultaneously experiences strong confinement and intense dot-impurity repulsive force. The diminished value of linear response at on and near off-centre locations indicates dominance of confining factors over the repulsive interaction. On the other hand, a far off-centre dopant undergoes marginal overlap with dot confinement centre. This makes the repulsive interaction insignificant and polarizability falls. The observed maximization at $r_{0} \sim 20.0$ a.u. reveals absolute dominance of factors that promote the dispersive nature of the system over the reverse ones. It is interesting to note that dopant location plays some important role in shaping the $\alpha_{x y}$ component in both the modes of application of noise. As a result we find a sudden surge in $\alpha_{x y}$ at a typical dopant location of $r_{0} \sim 20.0$ a.u. in both the cases. However, the very mode of application of noise affects the complete profile of $\alpha_{x y}$ component over the entire range of dopant site. We can thus infer that the additive and multiplicative nature of noise in general discriminates the relative dominance of diverse factors that control the dispersive character of the system as a function of dopant site. This is reflected through their overall distinct profiles; some kind of harmony, though, could be observed at some typical dopant site. The $\alpha_{y x}$ component exhibits nearly similar behaviour and the plots are not presented.

Fig. (2) represents the similar plots for $\beta_{x y y}$ and $\beta_{y x x}$ components with additive and multiplicative noise. In case of additive noise both the components exhibit similar pattern of variation with $r_{0}$. The variation consists of two distinct maxima; one at $r_{0} \sim 10.0$ a.u. and the other at $r_{0} \sim 35.0$ a.u. The near maxima are found to be more prominent than the distant one. The above two components, however, exhibit different profiles under the influence of multiplicative noise. The $\beta_{x y y}$ component exhibits distinct maxima at $r_{0} \sim 20.0$ a.u. whereas the $\beta_{y x x}$ component has been found to increase monotonically with $r_{0}$ up to $\sim 30.0$ a.u. beyond which it saturates with further shift of dopant. The emergence of second order polarizability bears close connection with the asymmetric as well as dispersive nature of the system. Since multiplicative noise undergoes direct coupling with the system coordinates, it interplays more delicately with the effective confinement of doped system in comparison with the additive counterpart. The difference in the overall behaviour of $\beta_{x y y}$ and $\beta_{y x x}$ components with variation of $\mathrm{r}_{0}$ thus naturally becomes highly conspicuous in case of multiplicative noise which can discriminate nonlinear polarizability components on the ba- 

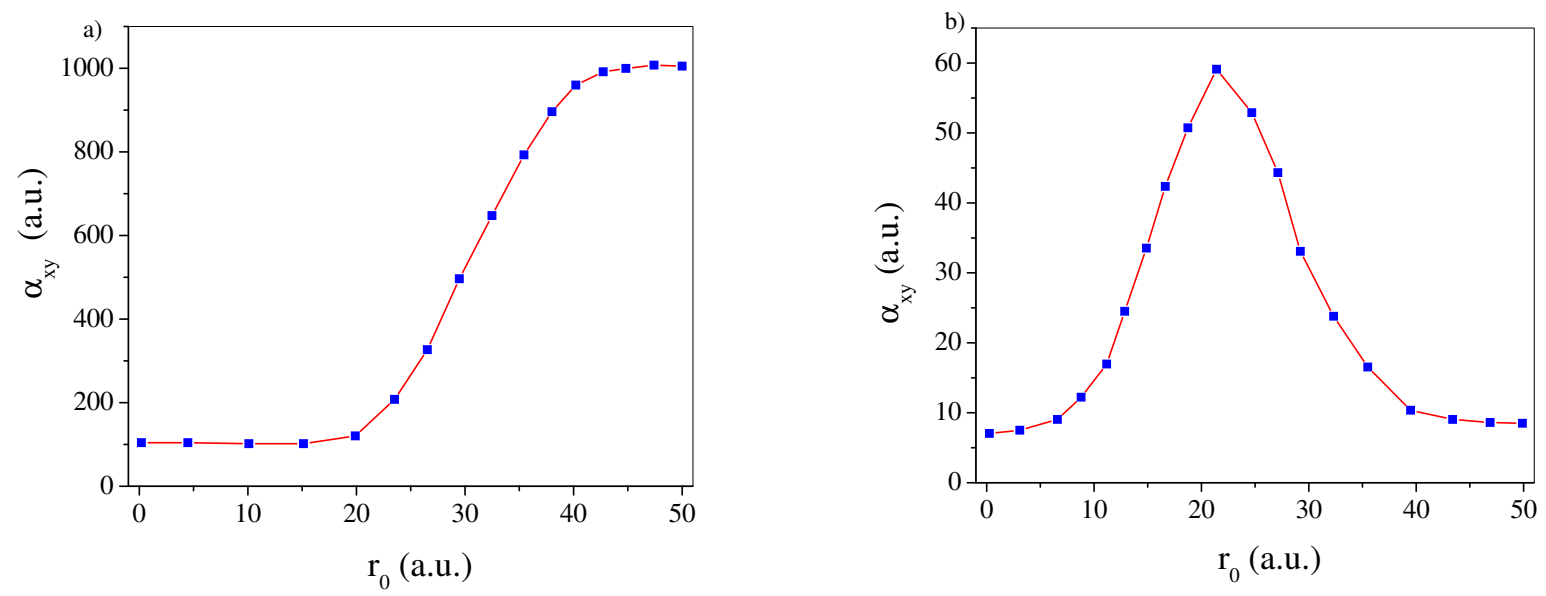

Fig. (1). Plot of $\alpha_{x y}$ components vs. $r_{0}$ : (a) for additive noise and (b) for multiplicative noise.

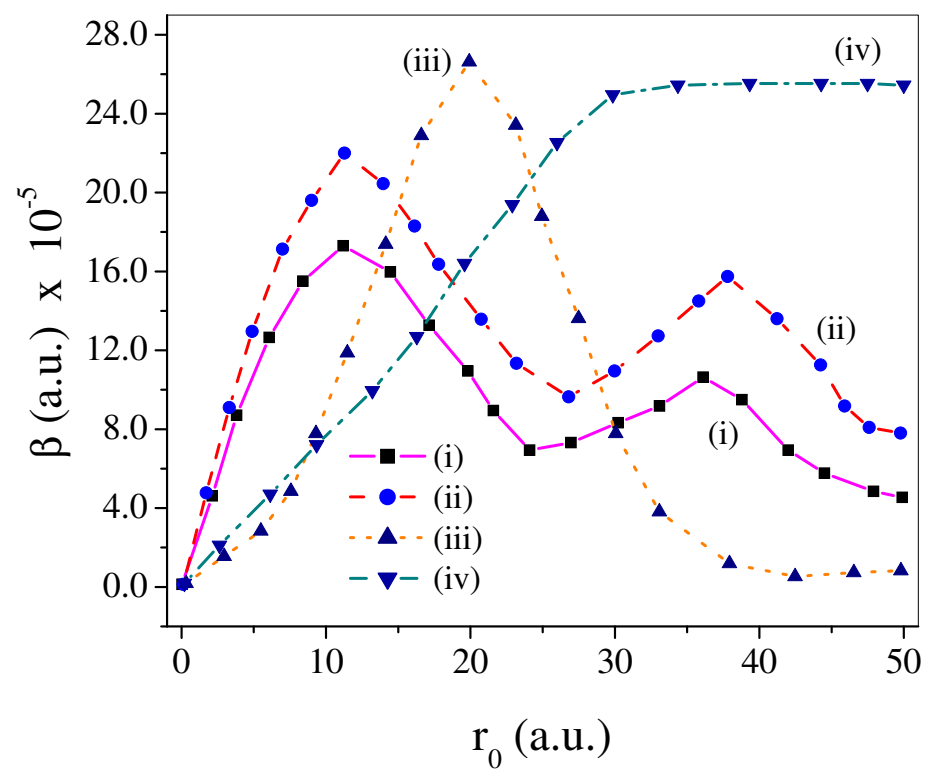

Fig. (2). Plot of $\beta$ components versus $r_{0}$ : (i) $\beta_{x y y}$ with additive noise, (ii) $\beta_{y x x}$ with additive noise, (iii) $\beta_{x y y}$ with multiplicative noise, (ii) $\beta_{y x x}$ with multiplicative noise.

sis of direction of applied field. It thus seems logical to realize that the lack of attachment of additive noise to system coordinates gives rise to nearly similar behaviour of the two components. Furthermore, the effect of gradual shift of dopant away from the dot confinement origin is not at all streamline. Such a shift makes the system more labile and facilitates emergence of the nonlinear polarizability. However, on the other hand, such a shift reduces dot-impurity repulsive interaction that could suppress polarizability. Hence such a shift of dopant basically causes an alteration in the effective confinement of the doped system and increases its asymmetric character. The expressions of various offdiagonal components [cf. eqn (12-14)] clearly reflect that, unlike $\alpha$ and $\gamma$, the $\beta$ components are non-equivalent.

Noise, by virtue of its basic nature makes the system more scattered. However, the scenario becomes quite complicated as the mode of application of noise simultaneously combines with various factors of relevance accompanying the shift of dopant site. The varying effective confinement, asymmetric, and dispersive nature of the system, mingle with noise and bring about a rich variety in the profiles of $\beta$ components. The profiles contain maximization and saturation of $\beta$ components as important ingredients at typical dopant sites.

Fig. (3a and 3b) delineate the plots of $\gamma_{x x y y}$ component with $r_{0}$ using additive and multiplicative noise, respectively. In case of additive noise we find a distinct minimum at $r_{0} \sim 22.0$ a.u. Fig. (3a). Application of multiplicative noise completely reverses the outcome and the said component displays maximization nearly at the same dopant location Fig. (3b). Thus, a change in the mode of noise application in turn causes a maximum reversal in the relative dominance of various parameters that promote or impede third order polarizability. The dopant location corresponding to this maxi- 

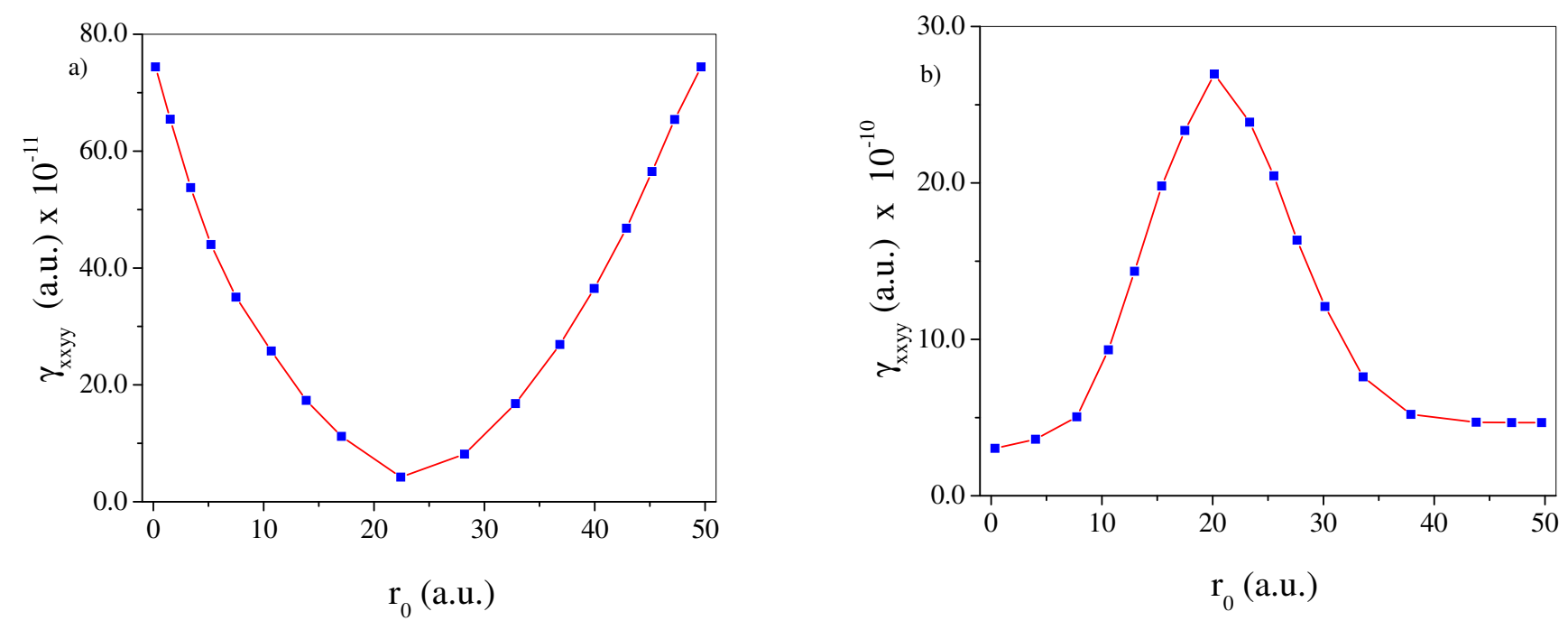

Fig. (3). Plot of $\gamma_{x x y y}$ components versus $r_{0}$ : (a) for additive noise and (b) for multiplicative noise.

mum reversal, however, remains nearly unchanged. The $\gamma_{\text {yyxx }}$ component depicts nearly similar feature (figures not shown).

The polarizability profiles discussed so far as function of $r_{0}$ strongly indicate that the dot-dopant interaction (or in other words the 'effective confinement potential') sincerely depends on $r_{0}$ and runs in conformity with the important observations of Karabulut and Baskoutas [20], and Baskoutas et al. [23] in related context. The dopant incorporated around a particular location undergoes typical interplay with dot confinement center. The mode of application of noise further enhances the delicacy of said typical interplay when it modulates the off-diagonal linear and nonlinear polarizability components in visibly different fashions. It needs to be further mentioned that, in agreement with our earlier works [31, 32] here also we do not find any influence of noise strength $(\mu)$ on the linear and nonlinear polarizabilities in case of additive noise. However, as before, the application of multiplicative noise invites noise strength dependence of polarizability components $[31,32]$. The present enquiry on off- diagonal components, however, reveals one striking contrast with our erstwhile findings on diagonal components in the presence of noise [31,32]. Previously we have envisaged tremendous enhancement of diagonal polarizability components by several orders of magnitude using multiplicative noise over that of additive analog $[31,32]$. Interestingly, in the present enquiry involving off-diagonal components, their magnitudes remain comparable in both the modes of application of noise. It thus appears that a change in the mode of application of noise to doped QD affects the diagonal components of linear and nonlinear polarizabilities much more severely than the off-diagonal counterparts.

\section{B. Role of Noise Strength}

Fig. (4a-d) depict the plots of $\alpha_{x y}, \beta_{x y y}, \beta_{y x x}$, and $\gamma_{x x y y}$ respectively, as functions of strength $(\mu)$ of multiplicative noise. The $\alpha_{x y}$ component increases monotonically to a maximum with increase in $\mu$ up to $\mu \sim 5.5 \times 10^{-7}$ a.u. Fig. (4a). An increase in $\mu$ enhances the dispersive nature of the system which results in monotonic increase of $\alpha$ component.

After maximization, $\alpha_{x y}$ decreases with $\mu$ till $\mu \sim 7.2 \mathrm{x}$ $10^{-7}$ a.u. Such a fall in the said component within the domain $5.5 \times 10^{-7}$ a.u. $\leq \mu \leq 7.2 \times 10^{-7}$ a.u. seems quite contrary to our expectation and may have some different background. Within this noise strength regime the strong systemnoise interaction appears to enhance the effective confinement. Because of this enhanced confinement the dispersive nature of the system could quench leading to a drop in the $\alpha_{x y}$ component. Beyond $\mu \sim 7.2 \times 10^{-7}$ a.u. the dispersive character again becomes quite pronounced and permanently overcomes confinement leading to monotonic increase of $\alpha_{x y} . \alpha_{y x}$ component displays nearly similar behaviour, as well.

Both $\beta_{x y y}$ and $\beta_{y x x}$ components consist of distinct maxima at $\mu \sim 3.1 \times 10^{-7}$ a.u. and $\mu \sim 2.3 \times 10^{-7}$ a.u., respectively (Figs. (4b-c)). The maximization of first nonlinear polarizability of QD devices is of utmost technological importance and the observed behaviour indicates development of maximum asymmetric character of the doped system at particular values of noise strength. Thus, although the two components display an overall similar behaviour as functions of $\mu$, they differ in the typical value of $\mu$ where maximization occurs and in the pattern of their fall after maximization. The two profiles also manifest onset of saturation at high noise strength regime (which is, however, much more pronounced for $\beta_{x y y}$ than $\beta_{y x x}$ ) and thus suggest kind of negotiation between noise and the effective confinement strength.

Fig. (4d) evinces the variation of $\gamma_{x x y y}$ with $\mu$ comprising of distinct maxima at $\mu \sim 3.5 \times 10^{-7}$ a.u. The observation indicates that the dispersive character of the system reaches its most at this typical value of noise strength. A departure from this typical value on either direction diminishes the 

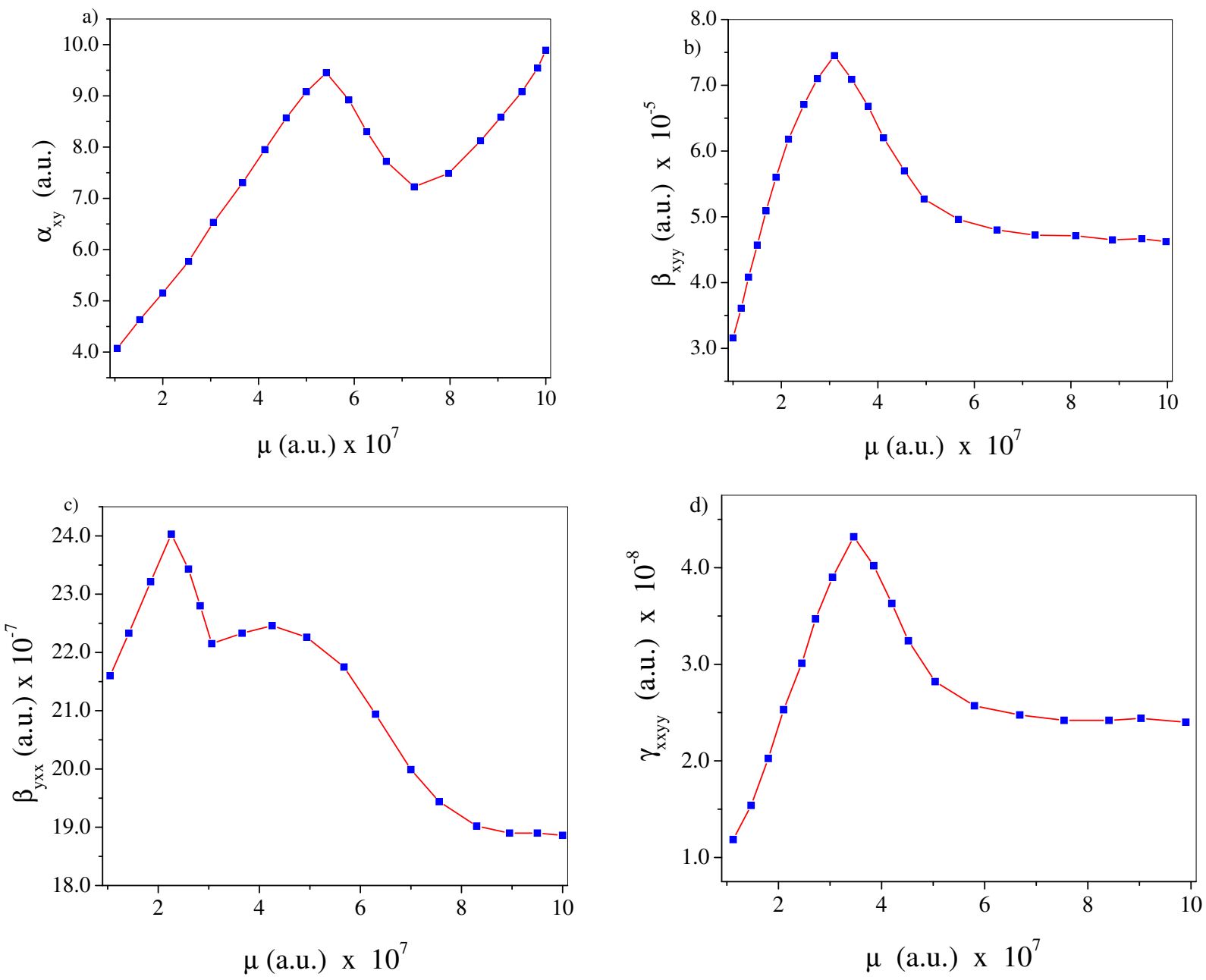

Fig. (4). Plot of off-diagonal polarizability components vs. $\mu$ with multiplicative noise: (a) for $\alpha_{x y}$, (b) for $\beta_{x y y}$, (c) for $\beta_{y x x}$, (d) for $\gamma_{x x y y}$.

dispersive character owing to the dominance of confinement effects over noise. The plot of $\gamma_{y y x x}$ are quite similar and not shown.

\section{CONCLUSION}

The off-diagonal components of static linear, first nonlinear and second nonlinear polarizabilities of impurity doped quantum dots have been investigated under the sway of Gaussian white noise. The polarizability components are found to be strongly dependent on the site of dopant incorporation and the mode of application (additive/multiplicative) of noise. A variation of dopant location or noise strength in turn affects the effective confinement of the system. Consequently, the dispersive and asymmetric character of the system are also affected. Whereas the off-diagonal $\alpha$ and $\gamma$ components mostly depend on dispersive character, the $\beta$ component also depends on the asymmetric character of the doped system. Particularly, the findings of the third order nonlinear polarizability show some connection with the notable works of Şahin [13] and Karabulut and Baskoutas [20]. It is because of its direct coupling to the system coordinates the multiplicative noise brings about more subtlety in the observed profiles of above polarizability components than its additive counterpart. In case of additive noise the noise strength remains indifferent to the polarizability components. Interestingly, unlike diagonal components [31,32], here the magnitudes of polarizability components remain comparable independent of the mode of application of noise. The present study also predicts a stronger influence of mode of application of noise on the diagonal polarizability components relative to off-diagonal ones. As a result of complex interplay between various pertinent parameters frequently we envisage maximization and saturation in the linear and nonlinear polarizability components that are driven by white noise signalling possibility of significant technological importance.

\section{CONFLICT OF INTEREST}

The authors confirm that this article content has no conflicts of interest.

\section{ACKNOWLEDGEMENTS}

Declared none. 


\section{REFERENCES}

[1] Gülveren B, Atav Ü, Sahin M, Tomak M. A parabolic quantum dot with N electrons and an impurity. Physica E 2005; 30: 143-9.

[2] Tas H, Sahin M. The electronic properties of core/shell/well/shell spherical quantum dot with and without a hydrogenic impurity. J Appl Phys 2012; 111: 083702.

[3] Baskoutas S, Terzis AF, Voutsinas E. Binding energy of donor states in a quantum dot $\mathrm{h}$ parabolic confinement. J Comput Theor Nanosci 2004; 1: 317-21.

[4] Sadeghi E, Avazpour A. Binding energy of an off-center donor impurity in ellipsoidal quantum dot with parabolic confinement potential. Physica B 2011; 406: 241-4.

[5] Barati M, Vahdani MRK, Rezaei G. Lower-lying states of hydrogenic impurity in lens-shaped and semi-lens-shaped quantum dots. J Phys: Condensed Matter 2007; 19: 136208.

[6] Yakar Y, Çakir B, Özmen A. Off-center hydrogenic impurity in spherical quantum dot with parabolic potential. Superlattice Microst 2013; 60: 389-97.

[7] Akgül S, Şahin M, KÖksal K. A detailed investigation of the electronic properties of a multilayer spherical quantum dot with a parabolic confinement. J Lumin 2012; 132: 1705-13.

[8] Vahdani MRK, Rezaei G. Linear and nonlinear optical properties of a hydrogenic donor in lens-shaped quantum dots. Phys Lett A 2009; 373: 3079-84.

[9] Xie W. Impurity effects on optical property of a spherical quantum dot in the presence of an electric field. Physica B 2010; 405: 3436440 .

[10] Karabulut I, Baskoutas S. Linear and nonlinear optical absorption coefficients and refractive index changes in spherical quantum dots: Effects of impurities, electric field, size, and optical intensity. J Appl Phys 2008; 103: 073512.

[11] Yakar Y, Çakir B, Özmen A. Calculation of linear and nonlinear optical absorption coefficients of a spherical quantum dot with parabolic potential. Opt Commun 2010; 283: 1795-800.

[12] Chen T, Xie W, Liang S. Optical and electronic properties of a two-dimensional quantum dot with an impurity. J Lumin 2013; 139: 64-8.

[13] Sahin M. Third-order nonlinear optical properties of a one- and two-electron spherical quantum dot with and without a hydrogenic impurity. J Appl Phys 2009; 106: 063710.

[14] Çakir B, Yakar Y, Özmen A, Sezer MÖ, Şahin M. Linear and nonlinear optical absorption coefficients and binding energy of a spherical quantum dot. Superlattice Microst 2010; 47: 556-66.

[15] Xie W. Nonlinear optical properties of a hydrogenic donor quantum dot. Phys Lett A 2008; 372: 5498-500.

[16] Baskoutas S, Paspalakis E, Terzis AF. Effects of excitons in nonlinear optical rectification in semiparabolic quantum dots. Phys Rev B 2006; 74: 153306.

[17] Zeng Z, Garoufalis CS, Terzis AF, Baskoutas S. Linear and nonlinear optical properties of $\mathrm{ZnS}-\mathrm{ZnO}$ core shell quantum dots: Effect of shell thickness, impurity, and dielectric environment. J Appl Phys 2013; 114: 023510.

[18] Peter AJ. Polarizabilities of shallow donors in spherical quantum dots with parabolic confinement. Phys Lett A 2006; 355: 59-62.

[19] Xie W. Linear and nonlinear optical properties of a hydrogenic donor in spherical quantum dots. Physica B 2008; 403: 4319-322.

[20] Karabulut I, Baskoutas S. Second and third harmonic generation susceptibilities of spherical quantum dots: effects of impurities, electric field and size. J Comput Theor Nanosci 2009; 6: 153-6.
[21] Kumar KM, Peter AJ, Lee CW. Optical properties of a hydrogenic impurity in a confined $Z_{1-x} C d_{x} S e=Z n S e$ quantum dot. Superlattices Microst 2012; 51: 184-93.

[22] Tiutiunnyk A, Tulupenko V, Mora-Ramos ME, Kasapoglu E, Ungan F, Sari H, SÖkmen I, Duque CA. Electron-related optical responses in triangular quantum dots. Physica E 2014; 60: 127-32.

[23] Baskoutas S, Paspalakis E, Terzis AF. Electronic structure and nonlinear optical rectification in a quantum dot: effects of impurities and external electric field. J Phys: Cond Mat 2007; 19: 395024 (9-pages).

[24] Murillo G, Porras-Montenegro N. Effects of an electric field on the binding energy of a donor impurity in a spherical $\mathrm{GaAs}(\mathrm{Ga} ; \mathrm{Al}) \mathrm{As}$ Quantum Dot with Parabolic Confinement. Phys Status Solidi B 2000; 220: 18790.

[25] Kirak M, Yilmaz S, Şahin M, Gencasian M. The electric field effects on the binding energies and the nonlinear optical properties of a donor impurity in a spherical quantum dot. J Appl Phys 2011; 109: 094309.

[26] Duque CM, Barseghyan MG, Duque CA. Donor impurity in vertically-coupled quantum dots under hydrostatic pressure and applied electric field. Eur Phys J B 2010; 73: 309-19.

[27] Mora-Ramos ME, Duque CA, Kasapoglu E, Sari H, SÖkmen I. Study of direct and indirect exciton states in $G a A s G a_{1-x} A l_{x} A s$ quantum dots under the effects of intense laser field and applied electric field. Eur Phys J B 2012; 85: 312.

[28] Narayanan M, Peter AJ. Electric field induced exciton binding energy and its non-linear optical properties in a narrow InSb/InGa $\mathrm{Sb}_{1-\mathrm{x}}$ quantum dot. Superlattice Microst 2012; 51: 48696.

[29] López SY, Porras-Montenegro N, Duque CA. Excitons in coupled quantum dots: hydrostatic pressure and electric field effects. Phys Status Solid B 2009; 246: 630-4.

[30] Kasapoglu E, Ungan F, Sari H, SÖkmen I, Mora-Ramos ME, Duque CA. Donor impurity states and related optical responses in triangular quantum dots under applied electric field. Superlattice Microst 2014; 73: 171-84.

[31] Ganguly J, Ghosh M. Influence of Gaussian white noise on the frequency-dependent linear polarizability of doped quantum dot. Chem Phys 2014; 438: 75-82.

[32] Ganguly J, Ghosh M. Influence of Gaussian white noise on the frequency-dependent first nonlinear polarizability of doped quantum dot. J Appl Phys 2014: 115; 174313.

[33] Ganguly J, Ghosh M. Exploring static and frequency-dependent third nonlinear polarizability of doped quantum dots driven by Gaussian white noise. Phys Status Solid B. DOI $10.1002 / \mathrm{pssb} .201451374$

[34] Adamowski J, Kwaśniowski A, Szafran B. LO-phonon-induced screening of electron electron interaction in $\mathrm{D}^{-}$centres and quantum dots. J Phys: Cond Mat 2005; 17: 4489-500.

[35] Bednarek S, Szafran B, Lis K, Adamowski J. Modeling of electronic properties of electrostatic quantum dots. Phys Rev B 2003; 68: 155333.

[36] Szafran B, Bednarek S, Adamowski J. Parity symmetry and energy spectrum of excitons in coupled self-assembled quantum dots. Phys Rev B 2001; 64: 125301.

[37] Gharaati A, Khordad R. A new confinement potential in spherical quantum dots: Modified Gaussian potential. Superlattice Microst 2010; 48: 276-87.

[38] Khordad R. Use of modified Gaussian potential to study an exciton in a spherical Quantum dot. Superlattice Microst 2013; 54: 7-15.

Received: November 24, 2014

(C) Saha et al.; Licensee Bentham Open.

This is an open access article licensed under the terms of the Creative Commons Attribution Non-Commercial License (http://creativecommons.org/licenses/by-nc/3.0/) which permits unrestricted, non-commercial use, distribution and reproduction in any medium, provided the work is properly cited. 